\title{
Superdiffusivity of asymmetric exclusion process in dimensions one and two
}

\author{
C. Landim*, J. Quastel† M. Salmhofer $\ddagger$ and H.-T. Yau ${ }^{\S}$ \\ IMPA, University of Toronto, Max-Planck-Institut, Leipzig, and Courant Institute
}

October 31, 2018

\begin{abstract}
We prove that the diffusion coefficient for the asymmetric exclusion process diverges at least as fast as $t^{1 / 4}$ in dimension $d=1$ and $(\log t)^{1 / 2}$ in $d=2$. The method applies to nearest and non-nearest neighbor asymmetric exclusion processes.
\end{abstract}

\section{Introduction}

Asymmetric exclusion is a Markov process on $\{0,1\}^{\mathbb{Z}^{d}}$ consisting of interacting continuous time random walks with asymmetric jump rates. There is at most one particle allowed per site. A particle at a site $x$ waits for an exponential time and then jumps to $y$ provided the site is not occupied. Otherwise the jump is suppressed and the process starts again. The jump is attempted at rate $p(y-x)$. In this article the jump law $p(\cdot)$ is assumed to have a nonzero mean, so that there is transport of the system.

Consider the system in equilibrium with a Bernoulli product measure of density $\rho$ as the invariant measure. Define the time dependent correlation function in equilibrium by

$$
S(x, t)=\left\langle\eta_{x}(t) ; \eta_{0}(0)\right\rangle
$$

If we choose $\rho=1 / 2$, there is no net global drift, i.e., $\sum_{x} x S(x, t)=0$. Otherwise one needs to subtract a net drift, which complicates but does not change the results or methods. Our main question is the behaviour for large $t$ of the diffusion coefficient,

$$
D(t)=\frac{1}{4 t} \sum_{x} x^{2} S(x, t) .
$$

In dimensions $d \geq 3$, the diffusion coefficient was proved to be bounded [8] for general asymmetric simple exclusion processes. Based on mode coupling theory, Beijeren, Kutner and Spohn [3] conjectured that $D(t) \sim(\log t)^{2 / 3}$ in dimension $d=2$ and $D(t) \sim t^{1 / 3}$ in

\footnotetext{
*Partially supported by CNRS, UMR 6085, landim@impa.br

${ }^{\dagger}$ Partially supported by NSERC, quastel@math.toronto.edu

${ }^{\ddagger}$ mns@mis.mpg.de

§Partially supported by NSF grant DMS-0072098, yau@cims.nyu.edu
} 
$d=1$. Similar predictions we made in [6] for the Kardar-Parisi-Zhang equation in $d=1$, which, when differentiated and appropriately discretized, yields the asymmetric exclusion process.

This problem has received much attention recently in the context of integrable systems. The main quantity analyzed there is fluctuation of the current across the origin in $d=1$ for the totally asymmetric simple exclusion process (only nearest neighbor jumps to the right), starting from the special initial configuration with all sites to the left of the origin occupied and all sites to the right of the origin empty. Johansson [5] observed that in this special situation the current across the origin can be mapped into a last passage percolation problem. By analyzing this problem asymptotically, Johansson proved that the variance of the current is of order $t^{2 / 3}$. In the case of discrete time, Baik and Rains [2] analyze an extended version of the last passage percolation problem and obtain fluctuations of order $t^{\alpha}$, where $\alpha=1 / 3$ or $\alpha=1 / 2$ depending on the parameters of the model. Both the approaches of [5] and [2] are related to the earlier results of Baik-Deift-Johansson [1] on the distribution of the length of the longest increasing subsequence in random permutations.

In [10] (see also [11]), Prähofer and Spohn succeeded in mapping the current of the totally asymmetric simple exclusion process into a last passage percolation problem for a general class of initial data, including the equilibrium case considered in this article. For the discrete time case, the extended problem is closely related to the work [2], but the boundary conditions are different. For continuous time, besides the boundary condition issue, one needs to extend the result of [2] from the geometric to the exponential distribution.

To relate these results to our problem, the variance of the current across the origin is proportional to

$$
\sum_{x}|x| S(x, t)
$$

Therefore, Johansson's result on the variance of the current can be interpreted as the spreading of $S(x, t)$ being of order $t^{2 / 3}$. If we combine the work of [10] and [2], neglect various issues discussed above, and extrapolate to the second moment, we obtain a growth of the second moment as $t^{4 / 3}$, consistent with the conjectured $D(t) \sim t^{1 / 3}$.

The results based on integrable systems give not just the variance of the current across the origin, but also its limiting distribution. The main restrictions appear to be the rigid requirements on the fine details of the dynamics and on the initial data, the restriction to one space dimension, and the special quantities which can be analysed. In fact, even for the totally asymmetric simple exclusion process in $d=1$ there was previously no proof that $D(t)$ diverges as $t \rightarrow \infty$. And for general asymmetric exclusion processes all of these problems were completely open. In this article, we present a method to study the diffusion coefficient of general asymmetric exclusion processes. Using this we obtain without too much work lower bounds $D(t) \geq(\log t)^{1 / 2}$ in dimension $d=2$ and $D(t) \geq t^{1 / 4}$ in $d=1$. We have restricted the proof to the case $\rho=1 / 2$, but a similar proof works for all densities away from zero or one.

\subsection{The model and main results}

Denote the particle configuration by $\eta=\left\{\eta_{x}\right\}_{x \in \mathbb{Z}^{d}}$ where $\eta_{x}$ is equal to 1 if site $x$ is occupied and is equal to 0 otherwise. Denote by $\eta^{x, y}$ the configuration obtained from $\eta$ by exchanging 
the occupation variables at $x$ and $y$ :

$$
\left(\eta^{x, y}\right)_{z}= \begin{cases}\eta_{z} & \text { if } z \neq x, y \\ \eta_{x} & \text { if } z=y \text { and } \\ \eta_{y} & \text { if } z=x\end{cases}
$$

We assume that the jump law $p(\cdot)$ is local, $p(z)=0$ for $|z| \geq L$ for some $L<\infty$, and that there is transport of mass, $\sum_{z} z p(z) \neq 0$. The generator of the asymmetric simple exclusion process is given by

$$
(\mathcal{L} f)(\eta)=\sum_{x, y \in \mathbb{Z}^{d}} p(y-x) \eta_{x}\left(1-\eta_{y}\right)\left[f\left(\eta^{x, y}\right)-f(\eta)\right] .
$$

For each $\rho$ in $[0,1]$, denote by $\nu_{\rho}$ the Bernoulli product measure on $\{0,1\}^{\mathbb{Z}^{d}}$ with density $\rho$ and by $\langle\cdot, \cdot\rangle_{\rho}$ the inner product in $L^{2}\left(\nu_{\rho}\right)$. The probability measures $\nu_{\rho}$ are invariant for the process.

For two cylinder functions $f, g$ and a density $\rho$, denote by $\langle f ; g\rangle_{\rho}$ the covariance of $f$ and $g$ with respect to $\nu_{\rho}$ :

$$
\langle f ; g\rangle_{\rho}=\langle f g\rangle_{\rho}-\langle f\rangle_{\rho}\langle g\rangle_{\rho} .
$$

Let $P_{\rho}$ denote the law of the asymmetric exclusion process starting from the equilibrium measure $\nu_{\rho}$. Expectation with respect to $P_{\rho}$ is denoted by $E_{\rho}$. Let

$$
S_{\rho}(x, t)=E_{\rho}\left[\left\{\eta_{x}(t)-\eta_{x}(0)\right\} \eta_{0}(0)\right]
$$

denote the time dependent correlation functions in equilibrium with density $\rho$. Denote by $\chi$ the compressibility given by

$$
\chi=\chi(\rho)=\sum_{x}\left\langle\eta_{x} ; \eta_{0}\right\rangle_{\rho}
$$

In our setting, $\chi(\rho)=\rho(1-\rho)$.

The bulk diffusion coefficient is defined by

$$
D_{i, j}(\rho, t)=\frac{1}{t} \frac{1}{2 \chi}\left\{\sum_{x \in \mathbb{Z}^{d}} x_{i} x_{j} S_{\rho}(x, t)-\chi\left(v_{i} t\right)\left(v_{j} t\right)\right\},
$$

where $v$ in $\mathbb{R}^{d}$ is the velocity defined by

$$
v t=\frac{1}{\chi} \sum_{x \in \mathbb{Z}^{d}} x E_{\rho}\left[\left\{\eta_{x}(t)-\eta_{x}(0)\right\} \eta_{0}(0)\right] .
$$

To simplify the notation we now specialize to the special case of the totally asymmetric simple exclusion process to the right in $d=1$ and, in $d=2$, jumps only to the nearest neighbor to the right in the $x_{1}$ coordinate, and jumps to both nearest neighbors in the $x_{2}$ coordinate with symmetric jump rule. More precisely, we take

$$
\begin{gathered}
(\mathcal{L} f)(\eta)=\sum_{x \in \mathbb{Z}} \eta_{x}\left(1-\eta_{x+1}\right)\left[f\left(\eta^{x, x+1}\right)-f(\eta)\right], \quad d=1 ; \\
(\mathcal{L} f)(\eta)=\sum_{x \in \mathbb{Z}^{2}} \eta_{x}\left[1-\eta_{x+e_{1}}\right]\left[f\left(\eta^{x, x+e_{1}}\right)-f(\eta)\right]+\frac{1}{2}\left[f\left(\eta^{x, x+e_{2}}\right)-f(\eta)\right], \quad d=2 .
\end{gathered}
$$


where we have combined the symmetric jump in the $x_{2}$-axis into the last term. We emphasize that the result and method in this paper apply to all asymmetric exclusion processes; the special choice is made only to simplify the notation. The velocity of the totally asymmetric simple exclusion process is explicitly computed as $v=2(1-2 \rho)$ in $d=1$ and $v=2(1-2 \rho) e_{1}$ in $d=2$. We further assume for simplicity that the density $\rho=1 / 2$ so that the velocity is zero.

Denote the instantaneous currents (i.e., the difference between the rate at which a particle jumps from $x$ to $x+e_{i}$ and the rate at which a particle jumps from $x+e_{i}$ to $x$ ) by $\tilde{w}_{x, x+e_{i}}$ :

$$
\tilde{w}_{x, x+e_{1}}=\eta_{x}\left[1-\eta_{x+e_{1}}\right], \quad \tilde{w}_{x, x+e_{2}}=\frac{1}{2}\left[\eta_{x+e_{2}}-\eta_{x}\right]
$$

We have the conservation law

$$
\mathcal{L} \eta_{0}+\sum_{i=1}^{d}\left(\tilde{w}_{-e_{i}, 0}-\tilde{w}_{0, e_{i}}\right)=0
$$

Let $w_{i}(\eta)$ denote the renormalized current in the $i$-th direction:

$$
w_{i}(\eta)=\tilde{w}_{0, e_{i}}-\left\langle\tilde{w}_{0, e_{i}}\right\rangle_{\rho}-\left.\frac{d}{d \theta}\left\langle\tilde{w}_{0, e_{i}}\right\rangle_{\theta}\right|_{\theta=\rho}\left(\eta_{0}-\rho\right)
$$

Note the subtraction of the linear term in this definition. We have

$$
w_{1}(\eta)=\left(\eta_{0}-\rho\right)\left(\eta_{e_{1}}-\rho\right)+\rho\left[\eta_{e_{1}}-\eta_{0}\right], \quad w_{2}(\eta)=\frac{1}{2}\left[\eta_{e_{2}}-\eta_{0}\right]
$$

A function $f$ on $\{0,1\}^{\mathbb{Z}^{d}}$ will be called local if it only depends on the variables at finitely many sites. For local functions $f$ and $g$ we define the semi-inner product

$$
\langle\langle g, h\rangle\rangle_{\rho}=\sum_{x \in \mathbb{Z}^{d}}<\tau_{x} g ; h>_{\rho}=\sum_{x \in \mathbb{Z}^{d}}<\tau_{x} h ; g>_{\rho} .
$$

Since the density $\rho$ is fixed to be $1 / 2$ in this article, we will henceforth leave out the subscript. All but a finite number of terms in this sum vanish because $\nu_{\rho}$ is a product measure and $g$, $h$ are local. From this inner product, we define the seminorm:

$$
\|f\|^{2}=\langle\langle f, f\rangle\rangle .
$$

Note that gradient terms $g=\tau_{x} h-h$ and all degree one functions vanish in this norm. Therefore we shall identify the currents $w$ with their degree two parts: For the rest of the article we set

$$
w_{1}(\eta)=\left(\eta_{0}-\rho\right)\left(\eta_{e_{1}}-\rho\right), \quad w_{2}(\eta)=0
$$

Fix a unit vector $\ell \in \mathbb{R}^{d}$. A simple calculation using Ito's formula [7] allows one to rewrite the diffusivity as

$$
\ell \cdot D(t) \ell-\frac{1}{2}=\frac{1}{\chi}\left\|t^{-1 / 2} \int_{0}^{t} \ell \cdot w(\eta(s)) d s\right\|^{2}
$$


This is a variant of the Green-Kubo formula [7]. In $d=1$ of course $D(t)$ is a scalar. In our special case in $d=2$, since $w_{2}=0, D(t)$ is a matrix with all entries zero except

$$
D_{11}(t)=\frac{1}{2}+\frac{1}{\chi}\left\|t^{-1 / 2} \int_{0}^{t} w_{1}(\eta(s)) d s\right\|^{2} .
$$

Recall that $\int_{0}^{\infty} e^{-\lambda t} f(t) d t \sim \lambda^{-\alpha}$ as $\lambda \rightarrow 0$ means, in some weak sense, that $f(t) \sim t^{\alpha-1}$ as $t \rightarrow \infty$. Throughout the following $\lambda$ will always be a positive real number. We can now state the main result.

Theorem 1 There exists $C>0$ so that for sufficiently small $\lambda>0$,

$$
\begin{aligned}
& d=1: \quad \int_{0}^{\infty} e^{-\lambda t} t D(t) d t \geq C \lambda^{-2-\frac{1}{4}} \\
& d=2: \quad \int_{0}^{\infty} e^{-\lambda t} t D_{11}(t) d t \geq C \lambda^{-2}|\log \lambda|^{1 / 2} .
\end{aligned}
$$

The conjectured behavior for $t$ large is $D(t) \sim t^{1 / 3}$ in $d=1$ and $D_{11}(t) \sim(\log t)^{2 / 3}$ in $d=2$. This theorem says that in a certain average, asymptotic sense $D(t) \geq t^{1 / 4}$ in $d=1$ and $D_{11}(t) \geq(\log t)^{1 / 2}$ in $d=2$.

From the definition, we can rewrite the diffusion coefficient as

$$
t D_{11}(t)=\frac{t}{2}+\frac{2}{\chi} \int_{0}^{t} \int_{0}^{s}\left\langle\left\langle e^{u \mathcal{L}} w_{1}, w_{1}\right\rangle\right\rangle d u d s
$$

in $d=2$, with an analogous formula in $d=1$ (just drop the subscripts). Thus

$$
\begin{aligned}
\int_{0}^{\infty} e^{-\lambda t} t D_{11}(t) d t & =\frac{1}{2 \lambda^{2}}+\frac{2}{\chi} \int_{0}^{\infty} d t \int_{0}^{t} \int_{0}^{s} e^{-\lambda t}\left\langle\left\langle e^{u \mathcal{L}} w_{1}, w_{1}\right\rangle\right\rangle d u d s \\
& =\frac{1}{2 \lambda^{2}}+\frac{2}{\chi} \int_{0}^{\infty} d u\left\{\int_{u}^{\infty} d t e^{-\lambda(t-u)}\left(\int_{u}^{t} d s\right)\right\}\left\langle\left\langle e^{-\lambda u} e^{u \mathcal{L}} w_{1}, w_{1}\right\rangle\right\rangle \\
& =\frac{1}{2 \lambda^{2}}+\chi^{-1} \lambda^{-2}\left\langle\left\langle w_{1},(\lambda-\mathcal{L})^{-1} w_{1}\right\rangle\right\rangle .
\end{aligned}
$$

Therefore, Theorem 1 follows from the following estimate on the resolvent.

Lemma 1.1 There exists $C>0$ such that for sufficiently small $\lambda>0$,

$$
\begin{array}{ll}
d=1: & \left\langle\left\langle w,(\lambda-\mathcal{L})^{-1} w\right\rangle\right\rangle \geq C \lambda^{-1 / 4} \\
d=2: & \left\langle\left\langle w_{1},(\lambda-\mathcal{L})^{-1} w_{1}\right\rangle\right\rangle \geq C|\log \lambda|^{1 / 2}
\end{array}
$$

\section{Duality and resolvent hierarchy}

Let $\mathcal{L}^{*}$ denote the adjoint of $\mathcal{L}$ with respect to the inner product of $L^{2}(\nu)$ and $\mathcal{S}=\left(\mathcal{L}+\mathcal{L}^{*}\right) / 2$ and $\mathcal{A}=\left(\mathcal{L}-\mathcal{L}^{*}\right) / 2$ be the symmetric and antisymmetric parts of $\mathcal{L}$ so that

$$
\mathcal{L}=\mathcal{S}+\mathcal{A} .
$$


Denote by $\mathcal{C}=\mathcal{C}(\rho)$ the space of $\nu_{\rho}$-mean zero local functions. For a finite subset $\Lambda$ of $\mathbb{Z}^{d}$, denote by $\xi_{\Lambda}$ the $\nu_{\rho}$-mean zero local function defined by

$$
\xi_{\Lambda}=\prod_{x \in \Lambda} \xi_{x}, \quad \xi_{x}=\frac{\eta_{x}-\rho}{\sqrt{\rho(1-\rho)}}
$$

Note that the collection $\left\{\xi_{\Lambda}\right\}$ where $\Lambda$ ranges over finite subsets of $\mathbb{Z}^{d}$, forms an orthonormal basis of $L^{2}\left(\nu_{\rho}\right)$. Denote by $\mathcal{M}_{n}$ the space of local functions of degree $n$, i.e., the space generated by monomials of degree $n$ :

$$
\mathcal{M}_{n}=\left\{f \in \mathcal{C} ; f=\sum_{|\Lambda|=n} f_{\Lambda} \xi_{\Lambda}, f_{\Lambda} \in \mathbb{R}\right\}
$$

Note that in the definition all but a finite number of coefficients $f_{\Lambda}$ vanish because $f$ is assumed to be local. Denote by

$$
\mathcal{C}_{n}=\cup_{1 \leq j \leq n} \mathcal{M}_{j}
$$

the space of cylinder functions of degree less than or equal to $n$. All $\nu_{\rho}$-mean zero local functions $f$ can be decomposed uniquely as a finite linear combination of cylinder functions of finite degree $: \mathcal{C}=\cup_{n \geq 1} \mathcal{M}_{n}$. Any $f \in L^{2}\left(\nu_{\rho}\right)$ can be written by degree,

$$
f=\left(f_{1}, f_{2}, f_{3}, \ldots\right)
$$

with $f_{n} \in \mathcal{M}_{n}$.

For $f \in \mathcal{M}_{n}$ represented as $f=\sum_{\Lambda,|\Lambda|=n} f_{\Lambda} \xi_{\Lambda}$ we have

$$
\|f\|^{2}=\sum_{n=1}^{\infty} \sum_{\Lambda \subset \mathbb{Z}^{d},|\Lambda|=n} \sum_{x \in \mathbb{Z}^{d}} f_{\tau_{x} \Lambda} f_{\Lambda} .
$$

Note that this effectively reduces the degree by one. A simple computation shows that in this basis the symmetric part $\mathcal{S}$ of $\mathcal{L}$ maps $\mathcal{M}_{n}$ into itself and is given by

$$
\mathcal{S} f(\eta)=-\frac{1}{2} \sum_{j=1}^{d} \sum_{x \in \mathbb{Z}^{d}} \sum_{\frac{\Omega,|\Omega|=n-1}{\Omega \cap\left\{x, x+e_{j}\right\}=\emptyset}}\left[f_{\Omega \cup\left\{x+e_{j}\right\}}-f_{\Omega \cup\{x\}}\right]\left[\xi_{\Omega \cup\left\{x+e_{j}\right\}}-\xi_{\Omega \cup\{x\}}\right] .
$$

The asymmetric part $\mathcal{A}$ can be decomposed into three pieces $\mathcal{A}=\mathcal{A}_{0}+\mathcal{A}_{+}-\mathcal{A}_{+}^{*}$ where $\mathcal{A}_{0}: \mathcal{M}_{n} \rightarrow \mathcal{M}_{n}$ into itself, $\mathcal{A}_{+}: \mathcal{M}_{n} \rightarrow \mathcal{M}_{n+1}$ and $\mathcal{A}_{+}^{*}: \mathcal{M}_{n} \rightarrow \mathcal{M}_{n-1}$ is the adjoint of $\mathcal{A}_{+}:$

$$
\begin{gathered}
\mathcal{A}_{0} f(\eta)=\frac{1-2 \rho}{2} \sum_{x \in \mathbb{Z}^{d}} \sum_{\frac{\Omega,|\Omega|=n-1}{\Omega \cap\left\{x, x+e_{1}\right\}=\emptyset}}\left[f_{\Omega \cup\left\{x+e_{1}\right\}}-f_{\Omega \cup\{x\}}\right]\left[\xi_{\Omega \cup\left\{x+e_{1}\right\}}+\xi_{\Omega \cup\{x\}}\right], \\
\mathcal{A}_{+} f(\eta)=-\sqrt{\rho(1-\rho)} \sum_{x \in \mathbb{Z}^{d}} \sum_{\frac{\Omega,|\Omega|=n-1}{\Omega \cap\left\{x, x+e_{1}\right\}=\emptyset}}\left[f_{\Omega \cup\left\{x+e_{1}\right\}}-f_{\Omega \cup\{x\}}\right] \xi_{\Omega \cup\left\{x, x+e_{1}\right\}} .
\end{gathered}
$$

In our special case $\rho=1 / 2$, we have $\mathcal{A}_{0}=0$ and thus $\mathcal{A}=\mathcal{A}_{+}-\mathcal{A}_{+}^{*}$. We can also identify $f=\sum_{\Lambda,|\Lambda|=n} f_{\Lambda} \xi_{\Lambda}$ with a symmetric function of $n$ variables,

$$
f\left(x_{1}, \ldots, x_{n}\right)= \begin{cases}f_{\left\{x_{1}, \ldots, x_{n}\right\}} & \text { if } x_{i} \neq x_{j} \text { for } i \neq j, i, j=1, \ldots, n \\ 0 & \text { otherwise }\end{cases}
$$


With this notation we have

$$
\begin{aligned}
\mathcal{A}_{+} f\left(x_{1}, \ldots, x_{n+1}\right) & =-\frac{1}{2} \sum_{i=1}^{n+1} \sum_{j \neq i} \delta\left(x_{j}-x_{i}-e_{1}\right) \prod_{k \neq j}\left(1-\delta\left(x_{j}-x_{k}\right)\right) \nabla_{+}^{i j} f \\
\nabla_{+}^{i j} f\left(x_{1}, \ldots, x_{n+1}\right) & =f\left(x_{1}, \ldots, x_{i}+e_{1}, \ldots, \widehat{x}_{j}, \ldots x_{n+1}\right)-f\left(x_{1}, \ldots, x_{i}, \ldots, \widehat{x_{j}}, \ldots, x_{n+1}\right)
\end{aligned}
$$

where $\delta(0)=1$ and zero otherwise, and $\widehat{x_{j}}$ indicates the absence of $x_{j}$ in the vector. Also,

$$
\begin{aligned}
\mathcal{S} f\left(x_{1}, \ldots, x_{n}\right) & =\sum_{i=1}^{n} \sum_{\sigma= \pm} \sum_{j=1}^{d} \prod_{k \neq i}\left(1-\delta\left(x_{i}+\sigma e_{j}-x_{k}\right)\right) \\
& \times\left[f\left(x_{1}, \ldots x_{i}+\sigma e_{j}, \ldots, x_{n}\right)-f\left(x_{1}, \ldots, x_{i}, \ldots, x_{n}\right)\right] .
\end{aligned}
$$

Note that $\mathcal{S}$ is the discrete Laplacian with Neumann boundary condition on

$$
\mathcal{E}_{1}=\left\{\mathbf{x}_{n}:=\left(x_{1}, \ldots, x_{n}\right): x_{i} \neq x_{j}, \text { for } i \neq j\right\} .
$$

The current $w \in \mathcal{C}_{2}$ and in our notation $w_{\left\{0, e_{1}\right\}}=1 / 4$ and $w_{\Lambda}=0$ for $\Lambda \neq\left\{0, e_{1}\right\},|\Lambda|=2$. The resolvent equation $(\lambda-\mathcal{L}) u=w$ becomes the hierarchy

$$
\begin{aligned}
\mathcal{A}_{+}^{*} u_{3}+(\lambda-\mathcal{S}) u_{2} & =w, \\
\mathcal{A}_{+}^{*} u_{k+1}+(\lambda-\mathcal{S}) u_{k}-\mathcal{A}_{+} u_{k-1} & =0, \quad k \geq 3 .
\end{aligned}
$$

The hierarchy starts at degree 2 instead of 1 because the $\langle\langle\cdot, \cdot\rangle\rangle$ inner product effectively reduces the degree by one. Any term $\mathcal{A}_{+} u_{1}$ is trivial in the sense of (2.5) and hence the degree one term plays no role and we can set $u_{1}=0$. In the same way, we disregard the degree one part of the current, and take $w_{\Lambda}=0$ for all finite subsets $\Lambda \subset \mathbb{Z}^{d}$ except $\left\{0, e_{1}\right\}$, and $w_{\left\{0, e_{1}\right\}}=1 / 4$.

Consider the truncated equation up to the degree $n$,

$$
\begin{aligned}
\mathcal{A}_{+}^{*} u_{3}+(\lambda-\mathcal{S}) u_{2} & =w, \\
\mathcal{A}_{+}^{*} u_{k+1}+(\lambda-\mathcal{S}) u_{k}-\mathcal{A}_{+} u_{k-1} & =0, \quad n-1 \geq k \geq 3 \\
(\lambda-\mathcal{S}) u_{n}-\mathcal{A}_{+} u_{n-1} & =0 .
\end{aligned}
$$

We can solve the final equation of (2.13) by

$$
u_{n}=(\lambda-\mathcal{S})^{-1} \mathcal{A}_{+} u_{n-1} .
$$

Substituting this into the equation of degree $n-1$, we have

$$
u_{n-1}=\left[(\lambda-\mathcal{S})+\mathcal{A}_{+}^{*}(\lambda-\mathcal{S})^{-1} \mathcal{A}_{+}\right]^{-1} u_{n-2} .
$$

Solving iteratively we arrive at

$$
u_{2}=\left[(\lambda-\mathcal{S})+\mathcal{A}_{+}^{*}\left\{(\lambda-\mathcal{S})+\cdots+\mathcal{A}_{+}^{*}\left((\lambda-\mathcal{S})+\mathcal{A}_{+}^{*}(\lambda-\mathcal{S})^{-1} \mathcal{A}_{+}\right)^{-1} \mathcal{A}_{+}\right\}^{-1} \mathcal{A}_{+}\right]^{-1} w
$$


Let $\pi_{n}: \mathcal{C} \rightarrow \mathcal{C}_{n}$ denote the projection onto degree $\leq n$ and $\mathcal{L}_{n}=\pi_{n} \mathcal{L} \pi_{n}$. The truncated equation represents the solution of $\left(\lambda-\mathcal{L}_{n}\right) u=w$ and hence $\left\langle\left\langle u_{2}, w\right\rangle\right\rangle=\left\langle\left\langle w,\left(\lambda-\mathcal{L}_{n}\right)^{-1} w\right\rangle\right\rangle$. For example

$$
\begin{array}{r}
\left\langle\left\langle w,\left[\lambda-\mathcal{S}+\mathcal{A}_{+}^{*}(\lambda-\mathcal{S})^{-1} \mathcal{A}_{+}\right]^{-1} w\right\rangle\right\rangle=\left\langle\left\langle w,\left(\lambda-\mathcal{L}_{3}\right)^{-1} w\right\rangle\right\rangle . \\
\left\langle\left\langle w,\left[\lambda-\mathcal{S}+\mathcal{A}_{+}^{*}\left\{\lambda-\mathcal{S}+\mathcal{A}_{+}^{*}(\lambda-\mathcal{S})^{-1} \mathcal{A}_{+}\right\}^{-1} \mathcal{A}_{+}\right]^{-1} w\right\rangle\right\rangle=\left\langle\left\langle w,\left(\lambda-\mathcal{L}_{4}\right)^{-1} w\right\rangle\right\rangle .
\end{array}
$$

Since $\mathcal{A}_{+}^{*}(\lambda-\mathcal{L})^{-1} \mathcal{A}_{+}$is nonnegative, we have the monotonicity inequality

$$
\begin{aligned}
\left\langle\left\langle w,\left(\lambda-\mathcal{L}_{3}\right)^{-1} w\right\rangle\right\rangle \leq & \left\langle\left\langle w,\left(\lambda-\mathcal{L}_{5}\right)^{-1} w\right\rangle\right\rangle \leq \cdots \leq\left\langle\left\langle w,(\lambda-\mathcal{L})^{-1} w\right\rangle\right\rangle \\
& \leq \cdots \leq\left\langle\left\langle w,\left(\lambda-\mathcal{L}_{4}\right)^{-1} w\right\rangle\right\rangle \leq\left\langle\left\langle w,\left(\lambda-\mathcal{L}_{2}\right)^{-1} w\right\rangle\right\rangle .
\end{aligned}
$$

To check that $\left\langle\left\langle w,(\lambda-\mathcal{L})^{-1} w\right\rangle\right\rangle$ is in fact the limit of these upper and lower bounds we use the variational formula. For any matrix $M$, let $M_{s}$ denote the symmetric part $\left(M+M^{*}\right) / 2$. The identity $\left\{\left[M^{-1}\right]_{s}\right\}^{-1}=M^{*}\left(M_{s}\right)^{-1} M$ always holds, and thus we have

$$
\left\langle\left\langle w,(\lambda-\mathcal{L})^{-1} w\right\rangle\right\rangle=\sup _{f}\left\{2\langle\langle w, f\rangle\rangle-\left\langle\left\langle(\lambda-\mathcal{L}) f,(\lambda-\mathcal{S})^{-1}(\lambda-\mathcal{L}) f\right\rangle\right\rangle\right\} .
$$

Note that

$$
\left\langle\left\langle(\lambda-\mathcal{L}) f,(\lambda-\mathcal{S})^{-1}(\lambda-\mathcal{L}) f\right\rangle\right\rangle=\langle\langle f,(\lambda-\mathcal{S}) f\rangle\rangle+\left\langle\left\langle\mathcal{A} f,(\lambda-\mathcal{S})^{-1} \mathcal{A} f\right\rangle\right\rangle .
$$

Hence

$$
\left\langle\left\langle w,(\lambda-\mathcal{L})^{-1} w\right\rangle\right\rangle=\sup _{f} \inf _{g}\left\{2\left\langle\left\langle w-\mathcal{A}^{*} g, f\right\rangle\right\rangle-\langle\langle f,(\lambda-\mathcal{S}) f\rangle\rangle+\langle\langle g,(\lambda-\mathcal{S}) g\rangle\rangle\right\} .
$$

Let $a_{n}$ denote the supremum restricted to $f \in \mathcal{C}_{n}$, and $a^{n}$ denote the infimum restricted to $g \in \mathcal{C}_{n}$ so that $a_{n} \uparrow\left\langle\left\langle w,(\lambda-\mathcal{L})^{-1} w\right\rangle\right\rangle$ and $a^{n} \downarrow\left\langle\left\langle w,(\lambda-\mathcal{L})^{-1} w\right\rangle\right.$. By straightforward computation one checks that $a_{n} \leq\left\langle\left\langle w,\left(\lambda-\mathcal{L}_{n}\right)^{-1} w\right\rangle\right\rangle \leq a^{n}$, giving the desired result.

In what follows we will present a general approach to the equations (2.13) which, from (2.15) and (2.14) give a nontrivial lower bound on the diffusion coefficient without too much work. Because it gives a sequence of upper and lower bounds, the method has the potential to give the full conjectured scaling of the diffusion coefficient.

\section{$3 \quad$ Degree 3 lower bounds}

From (2.15) and (2.14) of the previous section we have a lower bound at degree three. However, computations are complicated by the hard core exclusion. We now describe a method to remove the hard core restriction in the computation. We then perform the computations in Fourier space. The estimates which justify the removal of the hard core are presented in the next section.

Recall (2.10) the set $\mathcal{E}_{1}$ consists of configurations of $n$ particles, no two of which occupy the same site. In the previous section we consider functions on $\mathcal{E}_{1}$, and operators $\mathcal{S}$ and $\mathcal{A}_{+}$

acting on them. By removal of the hard core, we mean replacing these by functions on $\mathbb{Z}^{d n}$ and operators acting on these. 
Suppose that $f$ is a function of particle configurations $f_{\left\{x_{1}, \ldots, x_{n}\right\}},\left(x_{1}, \ldots, x_{n}\right) \in \mathcal{E}_{1}$. We can extend $f$ to all of $\mathbb{Z}^{n d}$ by defining $f\left(x_{1}, \ldots, x_{n}\right)=f_{\left\{x_{1}, \ldots, x_{n}\right\}}$ if $\left(x_{1}, \ldots, x_{n}\right) \in \mathcal{E}_{1}$ and $f\left(x_{1}, \ldots, x_{n}\right)=0$ otherwise. Note that

$$
E\left[\left|\sum_{|A|=n} f_{A} \xi_{A}\right|^{2}\right]=\frac{1}{n !} \sum_{x_{1}, \ldots, x_{n} \in \mathbb{Z}^{d}}\left|f\left(x_{1}, \ldots, x_{n}\right)\right|^{2}
$$

For a function $f: \mathbb{Z}^{n d} \rightarrow \mathbb{R}$, we shall use the same symbol $\langle f\rangle$ to denote the expectation

$$
\frac{1}{n !} \sum_{x_{1}, \ldots, x_{n} \in \mathbb{Z}^{d}} f\left(x_{1}, \ldots, x_{n}\right)
$$

and write the inner product of two functions as $\langle f, g\rangle=\langle f g\rangle$. If $f$ and $g$ vanish on $\mathcal{E}_{1}$, this coincides with the inner product introduced before. We also define, as before, $\langle\langle f, g\rangle\rangle=$ $\sum_{x \in \mathbb{Z}^{d}}\left\langle\tau_{x} f, g\right\rangle$. We use $\pi_{n}$ for the projection onto $\mathcal{C}_{n}$.

We now define $A_{+} F$ for symmetric functions not necessarily vanishing on $\mathcal{E}_{1}$ by a formula analogous to (2.8), except that we drop the product of delta functions:

$$
A_{+} F\left(x_{1}, \ldots, x_{n+1}\right)=\frac{1}{2} \sum_{i=1}^{n+1} \sum_{j \neq i} \delta\left(x_{j}-x_{i}-e_{1}\right) \nabla_{+}^{i j} F\left(x_{1}, \ldots, x_{n+1}\right)
$$

Notice that $\left\langle A_{+} F\right\rangle=0$ if $\langle F\rangle<\infty$ and hence the counting measure is invariant. The discrete Laplacian is given by

$$
\Delta F\left(x_{1}, \ldots, x_{n}\right)=\sum_{i=1}^{n} \sum_{\sigma= \pm} \sum_{\alpha=1,2}\left[F\left(x_{1}, \ldots x_{i}+\sigma e_{\alpha}, \ldots, x_{n}\right)-F\left(x_{1}, \ldots, x_{i}, \ldots, x_{n}\right)\right]
$$

and we define

$$
L=\Delta+A
$$

Let $L_{n}=\pi_{n} L \pi_{n}$ be the restriction of $L$ to $\mathcal{C}_{n}$.

Throughout the rest of the paper we will use $C(\lambda)$ to denote a function of $\lambda>0$ which has the property that for some $C<\infty$, and for sufficiently small $\lambda$,

$$
C(\lambda) \leq \begin{cases}C|\log \lambda|, & d=1 \\ C, & d=2\end{cases}
$$

In the next section we will prove the following lemma.

Lemma 3.1 There exists a $C(\lambda)$ as in (3.4) such that

$$
\frac{1}{C(\lambda) n^{2}}\left\langle\left\langle w,\left(\lambda-L_{n}\right)^{-1} w\right\rangle\right\rangle \leq\left\langle\left\langle w,\left(\lambda-\mathcal{L}_{n}\right)^{-1} w\right\rangle\right\rangle \leq C(\lambda) n^{2}\left\langle\left\langle w,\left(\lambda-L_{n}\right)^{-1} w\right\rangle\right\rangle .
$$

The special case $n=3$ combined with (2.15) and (2.14) tells us that

$$
\left\langle\left\langle w,\left[\lambda-\Delta+A_{+}^{*}(\lambda-\Delta)^{-1} A_{+}\right]^{-1} w\right\rangle\right\rangle \leq C(\lambda)\left\langle\left\langle w,(\lambda-\mathcal{L})^{-1} w\right\rangle\right\rangle .
$$


We define the Fourier transform of $F: \mathbb{Z}^{n d} \rightarrow \mathbb{R}$ by

$$
\widehat{F}\left(\mathbf{p}_{n}\right)=\sum_{\mathbf{x}_{n} \in \mathbb{Z}^{n d}} e^{-i \mathbf{x}_{n} \cdot \mathbf{p}_{n}} F\left(\mathbf{x}_{n}\right)
$$

for $\mathbf{p}_{n} \in\left(\mathbb{R}^{d} / 2 \pi \mathbb{Z}^{d}\right)^{n}$. The Fourier transform of the discrete Laplacian acting on $F$ is given by

$$
\widehat{-\Delta F}\left(\mathbf{p}_{n}\right)=-\sum_{j=1}^{n} \sum_{k=1}^{d}\left[e^{i e_{k} p_{j}}-2+e^{-i e_{k} p_{j}}\right] \hat{F}\left(p_{1}, \ldots, p_{n}\right)=\omega\left(\mathbf{p}_{n}\right) \hat{F}\left(\mathbf{p}_{n}\right)
$$

where $\omega\left(\mathbf{p}_{n}\right)=\sum_{j=1}^{n} \omega\left(p_{j}\right)$. In $d=2$ we will denote the $d$ components of $p$ by $(r, s)$ : $\mathbf{r}_{n}=\left(r_{1}, \ldots, r_{n}\right)$ and $\mathbf{s}_{n}=\left(s_{1}, \ldots, s_{n}\right)$. In $d=1, \omega(p)=-\left[e^{i p}-2+e^{-i p}\right]$ and in $d=2$, $\omega(p)=-\left[e^{i r}-2+e^{-i r}\right]-\left[e^{i s}-2+e^{-i s}\right]$. Note that in both cases $\omega$ is real valued and nonnegative. If $F$ is a symmetric function of two integer variables we have

$$
\widehat{A_{+} F}\left(p_{1}, p_{2}, p_{3}\right)=-\frac{1}{3 !} \sum_{\sigma}\left[e^{i e_{1} \cdot p_{\sigma_{1}}}-e^{-i e_{1} \cdot p_{\sigma_{3}}}\right] \hat{F}\left(p_{\sigma_{1}}+p_{\sigma_{3}}, p_{\sigma_{2}}\right)
$$

where $\sigma$ runs over permutations of degree three. By definition, we have

$$
\langle\langle F, G\rangle\rangle=\sum_{z} \int d p_{1} d p_{2} \hat{F}\left(p_{1}, p_{2}\right) \hat{G}\left(p_{1}, p_{2}\right) e^{i\left(p_{1}+p_{2}\right) z}=\int d p \hat{F}(p,-p) \hat{G}(p,-p)
$$

In other words, when considering the inner product $\langle\langle\cdot, \cdot\rangle\rangle$, we can consider the class of $\hat{F}\left(p_{1}, \cdots, p_{n}\right)$ defined only on the subspace $\sum_{j} p_{j}=0 \bmod 2 \pi \mathbb{Z}^{d}$.

Lemma 3.2 Suppose $F \in \mathcal{M}_{2}$. There exists a $C<\infty$ such that

$$
\begin{array}{ll}
d=1: & \left\langle\left\langle A_{+} F,(\lambda-\Delta)^{-1} A_{+} F\right\rangle\right\rangle \leq C \int_{u \in[-\pi, \pi)} \omega(u)[\lambda+\omega(u)]^{-1 / 2}|\hat{F}(u,-u)|^{2} d u ; \quad(3.7) \\
d=2: & \left\langle\left\langle A_{+} F,(\lambda-\Delta)^{-1} A_{+} F\right\rangle\right\rangle \leq C \int_{u \in[-\pi, \pi)^{2}} \omega\left(e_{1} \cdot u\right)|\log (\lambda+\omega(u))||\hat{F}(u,-u)|^{2} d u .
\end{array}
$$

Proof: Using the Schwarz inequality to bound the cross terms by the diagonal terms, we can bound $A_{+} F$ by

$$
\begin{aligned}
& \left\langle\left\langle A_{+} F,(\lambda-\Delta)^{-1} A_{+} F\right\rangle\right\rangle=\int_{p_{1}+p_{2}+p_{3}=0} \frac{\left|\widehat{A_{+} F}\left(p_{1}, p_{2}, p_{3}\right)\right|^{2}}{\lambda+\omega\left(p_{1}\right)+\omega\left(p_{2}\right)+\omega\left(p_{3}\right)} d S \\
& \leq C \int_{p_{1}+p_{2}+p_{3}=0} \frac{\left|e^{i e_{1} \cdot p_{1}}-e^{-i e_{1} \cdot p_{3}}\right|^{2}\left|\hat{F}\left(p_{1}+p_{3}, p_{2}\right)\right|^{2}}{\lambda+\omega\left(p_{1}\right)+\omega\left(p_{2}\right)+\omega\left(p_{3}\right)} d S
\end{aligned}
$$

where $d S$ is the element of surface area on the hyperplane $\left\{p_{1}+p_{2}+p_{3}=0\right\}$. Let $\Gamma$ denote the region in which at least one of the integration variables, $r_{i}$ or $s_{i}$ in $d=2$ or $p_{i}$ in $d=1$, is bounded away from $\pm \pi$ and 0 , let us say by $1 / 8$. On $\Gamma$ the denominator,

$$
\lambda+\omega\left(p_{1}\right)+\omega\left(p_{2}\right)+\omega\left(p_{3}\right) \geq C^{-1}>0
$$

independent of $\lambda$. Hence the integration over $\Gamma$ in (3.9) is uniformly bounded in $\lambda$. We are only concerned with terms diverging as $\lambda \downarrow 0$ and hence we can restrict our attention to the integration over $\Gamma^{C}$. 
We need to divide $\Gamma^{C}$ according to whether $p_{1}, p_{3}$ in $d=1$ and $r_{1}, r_{3}, s_{1}, s_{3}$ in $d=2$ are within $1 / 8$ of $-\pi=\pi$ or 0 . There are four regions in $d=1$ and sixteen in $d=2$. We have to compute each one and add them up. But in fact they are all analogous, and give the same result. So for simplicity we only present the region where they are all in $[-1 / 8,1 / 8]$.

We call $u=p_{1}+p_{3}, v=p_{1}-p_{3}$. The integration over corresponding region in (3.9) is bounded by a constant multiple of

$$
\int_{|u| \leq 1 / 8} \omega(u)|\hat{F}(u,-u)|^{2}\left\{\int_{|v| \leq 1 / 8} \frac{d v}{\lambda+|u+v|^{2}+|u-v|^{2}+|u|^{2}}\right\} d u
$$

in $d=1$ and

$$
\int_{\left|u \cdot e_{1}\right|,\left|u \cdot e_{2}\right| \leq 1 / 8} \omega\left(e_{1} \cdot u\right)|\hat{F}(u,-u)|^{2}\left\{\int_{\left|v \cdot e_{1}\right|,\left|v \cdot e_{2}\right| \leq 1 / 8} \frac{d v}{\lambda+|u+v|^{2}+|u-v|^{2}+|u|^{2}}\right\} d u
$$

in $d=2$. Estimating the inside integration, in brackets, in $d=1$,

$$
\int_{|v| \leq 1 / 8} \frac{d v}{\lambda+(u+v)^{2}+(u-v)^{2}+u^{2}} \leq C\left(\lambda+u^{2}\right)^{-1 / 2} .
$$

In $d=2$,

$$
\int_{\left|v \cdot e_{1}\right|,\left|v \cdot e_{2}\right| \leq 1 / 8} \frac{d v}{\lambda+|u+v|^{2}+|u-v|^{2}+|u|^{2}} \leq C\left|\log \left(\lambda+u^{2}\right)\right|,
$$

which completes the proof of the lemma.

Lemma 3.3 There exists $C<\infty$ such that

$$
\left\langle\left\langle w,\left[\lambda-\Delta+A_{+}^{*}(\lambda-\Delta)^{-1} A_{+}\right]^{-1} w\right\rangle\right\rangle \geq \begin{cases}C^{-1} \lambda^{-1 / 4} & d=1 ; \\ C^{-1}|\log \lambda|^{1 / 2} & d=2 .\end{cases}
$$

Proof: $\quad d=1$ : The Fourier transform of the current $w$ is

$$
\hat{w}\left(p_{1}, p_{2}\right)=e^{-i p_{2}} / 2
$$

From the previous lemma the left hand side of (3.10) is bounded below by

$$
C^{-1} \int_{-\pi}^{\pi} \frac{d \xi}{\lambda+\omega(\xi)(\lambda+\omega(\xi))^{-1 / 2}}
$$

We can restrict the integration to the region $\xi \in[-1 / 8,1 / 8]$ and replace $\omega(x)$ by $x^{2}$ to have a further lower bound,

$$
\int_{-1 / 8}^{1 / 8} \frac{d \xi}{\lambda+\xi^{2}\left(\lambda+\xi^{2}\right)^{-1 / 2}} \geq C^{-1} \lambda^{-1 / 4}
$$

This proves Lemma 1.1 for $d=1$.

$d=2$ : The Fourier transform of the current $w$ is

$$
\widehat{w}\left(p_{1}, p_{2}\right)=e^{-i r_{2} / 2}
$$


From the previous lemma the left hand side of (3.10) is bounded below by

$$
C^{-1} \int_{-\pi}^{\pi} \int_{-\pi}^{\pi} \frac{d \xi d \eta}{\lambda+\omega(\eta)+\omega(\xi)+\omega(\xi)|\log (\lambda+\omega(\eta)+\omega(\xi))|}
$$

We can restrict to the region $\xi, \eta \in[-1 / 8,1 / 8]$ to have a further lower bound. In this region, we can replace $\omega(x)$ by $x^{2}$ up to a constant factor and use $\left|\log \left(\lambda+\eta^{2}+\xi^{2}\right)\right| \leq\left|\log \left(\lambda+\eta^{2}\right)\right|$ to obtain a further lower bound

$$
C^{-1} \int_{-1 / 8}^{1 / 8} \int_{-1 / 8}^{1 / 8} \frac{d \xi d \eta}{\lambda+\eta^{2}+\xi^{2}+\xi^{2}\left|\log \left(\lambda+\eta^{2}\right)\right|}
$$

Letting $z=\xi\left(1+\left|\log \left(\lambda+\eta^{2}\right)\right|\right)^{1 / 2}$ the integration is bounded below by

$$
\int_{-1 / 8}^{1 / 8} \int_{-1 / 8}^{1 / 8} \frac{d z d \eta}{\lambda+\eta^{2}+z^{2}}\left(1+\log \left(\lambda+\eta^{2}\right) \mid\right)^{-1 / 2}
$$

Changing to polar coordinates $r, \theta$ and restricting to $\pi / 3 \leq \theta \leq 2 \pi / 3$ we get another lower bound,

$$
\int_{0}^{1 / 20} \frac{r d r}{\lambda+r^{2}}\left|\log \left(\lambda+r^{2}\right)\right|^{-1 / 2} \geq C^{-1}|\log \lambda|^{1 / 2} .
$$

Lemma 1.1 follows immediately from Lemma 3.3, (2.15) and Lemma 3.1 in $d=2$. However in $d=1$ this only gives the slightly weaker result $\left\langle\left\langle w(\lambda-\mathcal{L})^{-1}\right\rangle\right\rangle \geq C^{-1} \lambda^{-1 / 4} /|\log \lambda|$. The $\log \lambda$ is purely from the removal of the hard core, and we now show that the correct degree 3 lower bound in $d=1$ is of order $\lambda^{-1 / 4}$.

Proof of Lemma 1.1 in $d=1$ : We will choose a test function $f \in \mathcal{M}_{2}$ in (2.15) to obtain a lower bound. Because of the summation over shifts in the definition (1.9) of $\langle\langle\cdot, \cdot\rangle\rangle$ there is really a reduction in dimension from $\left\{\left(x_{1}, x_{2}\right) \in \mathbb{Z}^{2} \mid x_{2}>x_{1}\right\}$ to $\mathbb{Z}_{+}$. Hence we define for $x \in \mathbb{Z}_{+}$

$$
\bar{f}(x)=\sum_{y \in \mathbb{Z}} f(y, y+1+x) .
$$

Then we have

$$
\begin{gathered}
2\langle\langle w, f\rangle\rangle=\bar{f}(0), \\
\langle\langle f,(-\mathcal{S}) f\rangle\rangle=\frac{1}{2} \sum_{x \in \mathbb{Z}_{+}}[\bar{f}(x+1)-\bar{f}(x)]^{2},
\end{gathered}
$$

and if $\bar{g}\left(x_{1}, x_{2}\right)=\sum_{y} g\left(y, y+1+x_{1}, y+2+x_{2}\right)$,

$$
\overline{\mathcal{A}_{+} f}\left(x_{1}, x_{2}\right)=\delta\left(x_{1}\right)\left[\bar{f}\left(x_{2}-1\right)-\bar{f}\left(x_{2}\right)\right]+\delta\left(x_{2}\right)\left[\bar{f}\left(x_{1}+1\right)-\bar{f}\left(x_{1}\right)\right],
$$

and

$$
\langle\langle g,(-\mathcal{S}) g\rangle\rangle==\frac{1}{4} \sum_{x_{1}, x_{2} \in \mathbf{Z}_{+}} \sum_{v}\left(\bar{g}\left(\left(x_{1}, x_{2}\right)+v\right)-\bar{g}\left(x_{1}, x_{2}\right)\right)^{2}
$$

where $v$ runs over $\pm(1,0), \pm(0,1)$ and $\pm(-1,1)$ with addition intepreted as with reflecting boundary conditions at the origin. 
We now make the choice

$$
\bar{f}(x)=\lambda^{-1 / 4} e^{-\lambda^{3 / 4} x}
$$

We have

$$
\lambda \sum_{x \in \mathbb{Z}_{+}}|\bar{f}(x)|^{2}=\lambda^{1 / 2}\left(1-e^{-2 \lambda^{3 / 4}}\right)^{-1} \sim \frac{1}{2} \lambda^{-1 / 4}
$$

and

$$
\begin{gathered}
\sum_{x \in \mathbb{Z}_{+}}[\bar{f}(x+1)-\bar{f}(x)]^{2}=\lambda^{-1 / 2}\left(1-e^{-\lambda^{3 / 4}}\right) \sim \lambda^{1 / 4} \\
\overline{\mathcal{A}_{+} f}\left(x_{1}, x_{2}\right)=-\delta\left(x_{1}\right) \mathbf{1}\left(x_{2}>0\right) \lambda^{-1 / 4}\left(1-e^{\lambda^{3 / 4}}\right) e^{-\lambda^{3 / 4} x_{2}}+\delta\left(x_{2}\right) \lambda^{-1 / 4}\left(e^{-\lambda^{3 / 4}}-1\right) e^{-\lambda^{3 / 4} x_{1}} \\
\sim \lambda^{1 / 2}\left[\delta\left(x_{1}\right) \mathbf{1}\left(x_{2}>0\right) e^{-\lambda^{3 / 4} x_{2}}-\delta\left(x_{2}\right) e^{-\lambda^{3 / 4} x_{1}}\right] .
\end{gathered}
$$

and the final term $\left\langle\left\langle\mathcal{A}_{+} f,(\lambda-\mathcal{S})^{-1} \mathcal{A}_{+} f\right\rangle\right\rangle$ can be written

$$
\int_{0}^{\infty} d t e^{-\lambda t} \sum_{x_{1}, x_{2}, y_{1}, y_{2} \in \mathbf{Z}_{+}} p_{t}\left(\left(x_{1}, x_{2}\right),\left(y_{1}, y_{2}\right)\right) \overline{\mathcal{A}_{+} f}\left(x_{1}, x_{2}\right) \overline{\mathcal{A}_{+} f}\left(y_{1}, y_{2}\right)
$$

where $p_{t}$ are the transition probabilities of the random walk on $\mathbb{Z}_{+}^{2}$ with Dirichlet form given by (3.14). Using the small $\lambda$ approximation for $\overline{\mathcal{A}} f$ we get

$$
\lambda \int_{0}^{\infty} d t e^{-\lambda t} \sum_{x, y \in \mathbf{Z}_{+}} e^{-\lambda^{3 / 4}(x+y)}\left[(1+\mathbf{1}(x, y>0)) p_{t}((0, x),(0, y))-2 \mathbf{1}(x>0) p_{t}((0, x),(y, 0))\right] .
$$

By images we can rewrite this as

$$
\begin{aligned}
\lambda \int_{0}^{\infty} d t e^{-\lambda t} \sum_{x, y \in \mathbf{Z}} e^{-\lambda^{3 / 4}(|x|+|y|)}[ & (1+\mathbf{1}(|x|,|y|>0)) p_{t}((0, x),(0, y)) \\
& \left.-2 \mathbf{1}(x>0) p_{t}((0, x),(y, 0))\right]
\end{aligned}
$$

where $p_{t}$ is now the transition density for a continuous time random walk on $\mathbf{Z}^{2}$ where the particle makes jumps at rate $1 / 2$ of $(1,0),(-1,0),(0,1),(0,-1)$ and, in the first and third quadrants $(1,-1)$ and $(-1,1)$, and in the second and fourth quadrants $(1,1)$ and $(-1,-1)$. On the axes themselves the rules are changed a bit. For example, on the positive $x$-axis the particle jumps at rate $1 / 4$ of $(0,1),(0,-1),(-1,1)$ and $(-1,-1)$. On the other axes these rules are just naturally rotated. The diffusion approximation is

$$
2 \lambda \int_{0}^{\infty} d t e^{-\lambda t} \int_{-\infty}^{\infty} d x \int_{-\infty}^{\infty} d y e^{-\lambda^{3 / 4}(|x|+|y|)}\left[p_{t}((0, x),(0, y))-p_{t}((0, x),(y, 0))\right]
$$

where $p_{t}$ is now the transition density for a diffusion in $\mathbf{R}^{2}$ with generator $\Delta+d^{2}$ where $d=\partial_{y}-\partial_{x}$ in the first and third quadrants and $d=\partial_{y}+\partial_{x}$ in the second and fourth quadrants. The corresponding Dirichlet form is comparable to the standard one and therefore we can bound the transition probabilities above and below by those of Brownian motion (see (41). By change of variables

$$
\lambda \int_{0}^{\infty} d t e^{\lambda t} \int_{-\infty}^{\infty} d x \int_{-\infty}^{\infty} d y e^{-\lambda^{3 / 4}(|x+y|)} \frac{e^{-\frac{|y-x|^{2}}{4 t}}}{4 \pi t}=C \lambda^{-1 / 4}
$$


The error

$$
\lambda \int_{0}^{\infty} d t e^{\lambda t} \int_{-\infty}^{\infty} d x \int_{-\infty}^{\infty} d y\left[e^{-\lambda^{3 / 4}(|x|+|y|)}-e^{-\lambda^{3 / 4}(|x+y|)}\right] \frac{e^{-\frac{|y-x|^{2}}{4 t}}}{4 \pi t}=O(\lambda) .
$$

We can choose $\alpha \phi$ for our test function instead of $\phi$. In summary we have

$$
\left\langle\left\langle w,\left(\lambda-\mathcal{L}^{-1}\right) w\right\rangle\right\rangle \geq 2 \alpha \lambda^{-1 / 4}-C \alpha^{2} \lambda^{-1 / 4} \geq C^{\prime} \lambda^{-1 / 4}
$$

if $\alpha$ is chosen sufficiently small.

\section{Removal of hard core}

In this section we prove Lemma 3.1. Recall the main statement is that $\left\langle\left\langle w,\left(\lambda-\mathcal{L}_{n}\right)^{-1} w\right\rangle\right\rangle$ can be bounded above and below in terms of $\left\langle\left\langle w,\left(\lambda-L_{n}\right)^{-1} w\right\rangle\right\rangle$ at the expense of constants depending on $n$. Since we only use the bound for $n=3$ in this article, the precise dependence of the constants on $n$ is not important and in many places is probably not optimal.

For $f$ a symmetric function on $\mathbb{Z}^{n}$, we denote by $\rho_{i}, i=1,2,3$ the one, two and three point functions :

$$
\begin{aligned}
\rho_{1}(x) & =\frac{1}{(n-1) !} \sum_{x_{1}, \cdots, x_{n-1}}\left[f\left(x, x_{1}, \cdots, x_{n-1}\right)\right]^{2} \\
\rho_{2}(x, y) & =\frac{1}{(n-2) ! 2 !} \sum_{x_{1}, \cdots, x_{n-2}}\left[f\left(x, y, x_{1}, \cdots, x_{n-2}\right)\right]^{2} \\
\rho_{3}(x, y, z) & =\frac{1}{(n-3) ! 3 !} \sum_{x_{1}, \cdots, x_{n-3}}\left[f\left(x, y, z, x_{1}, \cdots, x_{n-3}\right)\right]^{2}
\end{aligned}
$$

for all $x \neq y \neq z$ in $\mathbb{Z}^{d}$. The following is Lemma 4.8 of 8 .

Lemma 4.1 Suppose $f$ is a symmetric function on $\mathbb{Z}^{n}$. Then we have in all dimensions

$$
\left\langle\rho_{3}^{1 / 2},\left(-\mathcal{S}_{3}\right) \rho_{3}^{1 / 2}\right\rangle \leq n^{2}\langle f,(-\mathcal{S}) f\rangle
$$

Proof: By symmetry,

$$
\left\langle\rho_{3}^{1 / 2},\left(-\mathcal{S}_{3}\right) \rho_{3}^{1 / 2}\right\rangle=3 \sum_{\sigma= \pm, e}\left\langle\left(\rho_{3}^{1 / 2}(x+\sigma e, y, z)-\rho_{3}^{1 / 2}(x, y, z)\right)^{2}\right\rangle
$$

where $e$ are summed over the standard basis of unit vectors in $\mathbb{R}^{n}$, and

$$
\langle f,(-\mathcal{S}) f\rangle=n \sum_{\sigma= \pm, e} \frac{1}{n !} \sum_{x, y, z, x_{1}, \ldots, x_{n-3}}\left[f\left(x+\sigma e, y, z, x_{1}, \ldots, x_{n-3}\right)-f\left(x, y, z, x_{1}, \ldots, x_{n-3}\right)\right]^{2} .
$$

By Schwarz's inequality, $\left(\left(\sum_{j} a_{j}^{2}\right)^{1 / 2}-\left(\sum_{j} b_{j}^{2}\right)^{1 / 2}\right)^{2} \leq \sum_{j}\left(a_{j}-b_{j}\right)^{2}$, we have

$$
\begin{aligned}
& \left(\rho_{3}^{1 / 2}(x, y, z)-\rho_{3}^{1 / 2}\left(x^{\prime}, y, z\right)\right)^{2} \\
& \leq \frac{1}{(n-3) ! 3 !} \sum_{x_{1}, \ldots, x_{n-3}}\left[f\left(x, y, z, x_{1}, \ldots, x_{n-3}\right)-f\left(x^{\prime}, y, z, x_{1}, \ldots, x_{n-3}\right)\right]^{2},
\end{aligned}
$$


and hence

$$
\begin{aligned}
& \left\langle\left(\rho_{3}^{1 / 2}(x+e, y, z)-\rho_{3}^{1 / 2}(x, y, z)\right)^{2}\right\rangle=\frac{1}{3 !} \sum_{x, y, z}\left(\rho_{3}^{1 / 2}(x, y, z)-\rho_{3}^{1 / 2}(x+e, y, z)\right)^{2} \\
& \leq \frac{1}{(n-3) !(3 !)^{2}} \sum_{x, y, z, x_{1}, \ldots, x_{n-3}}\left[f\left(x+e, y, z, x_{1}, \ldots, x_{n-3}\right)-f\left(x, y, z, x_{1}, \ldots, x_{n-3}\right)\right]^{2} .
\end{aligned}
$$

The following Lemma is a simple extension of Theorem 4.7 of [8] or Lemma 3.7 of [12 to dimensions $d=1,2$. Recall the definition (2.10) of $\mathcal{E}_{1}$.

Lemma 4.2 Fix $R>0$. There exist $C(\lambda)$ as in (3.4) such that for $f$ a symmetric function on $\mathbb{Z}^{n}$ vanishing on $\mathcal{E}_{1}^{C}$,

$$
\sum_{i \neq j \neq k}\left\langle 1_{\left\{\left|x_{i}-x_{k}\right|+\left|x_{k}-x_{j}\right| \leq R\right\}} f^{2}\right\rangle \leq C(\lambda) n^{2}\langle f,(\lambda-\mathcal{S}) f\rangle .
$$

Proof: By definition of the three point function, the left side is a constant times

$$
\left\langle 1_{\left\{\left|x_{1}-x_{3}\right|+\left|x_{3}-x_{2}\right| \leq R\right\}} \rho_{3}\left(x_{1}, x_{2}, x_{3}\right)\right\rangle
$$

By the previous Lemma, we can bound the Dirichlet form of $g=\rho_{3}^{1 / 2}$ by that of $f$. Thus we only have to prove that

$$
\left\langle 1_{\left\{\left|x_{1}-x_{3}\right|+\left|x_{3}-x_{2}\right| \leq R\right\}} g^{2}\right\rangle \leq C(\lambda)\langle g,(\lambda-\mathcal{S}) g\rangle
$$

for functions $g$ of degree three.

Recall that for configuration with three particles we have $\mathcal{E}_{1}=\left\{\mathbf{x}_{3}:=\left(x_{1}, x_{2}, x_{3}\right): x_{i} \neq\right.$ $x_{j}$, for $\left.i \neq j\right\}$. We have $g\left(\mathbf{x}_{3}\right)=0$ whenever $\mathbf{x}_{3} \notin \mathcal{E}_{1}$ and the operator $\mathcal{S}$ is the discrete Laplacian on $\mathcal{E}_{1}$ with Neumann boundary conditions. Define $G\left(\mathbf{x}_{3}\right)=g\left(\mathbf{x}_{3}\right)$ if $\mathbf{x}_{3} \in \mathcal{E}_{1}$ and $G\left(\mathbf{x}_{3}\right)=\operatorname{Av}_{\mathbf{y}_{3} \in \mathcal{E}_{1},\left|\mathbf{y}_{3}-\mathbf{x}_{3}\right| \leq 2} g\left(\mathbf{y}_{3}\right)$ for $\mathbf{x}_{3} \notin \mathcal{E}_{1}$. We claim that for $G$ so defined,

$$
\langle G(\lambda-\Delta) G\rangle \leq C\langle g(\lambda-\mathcal{S}) g\rangle
$$

Consider $\left\langle\left[G\left(\mathbf{x}_{3}\right)-G\left(x_{1}+e_{1}, x_{2}, x_{3}\right)\right]^{2}\right\rangle$ with $\left(x_{1}+e_{1}, x_{2}, x_{3}\right) \in \mathcal{E}_{1}$ and $\mathbf{x}_{3} \notin \mathcal{E}_{1}$. In this case that $G\left(\mathbf{x}_{3}\right)$ is the average of $g\left(\mathbf{y}_{3}\right)$ with $\left|\mathbf{y}_{3}-\mathbf{x}_{3}\right| \leq 2$ and $\mathbf{y}_{3} \in \mathcal{E}_{1}$. We can check that $\mathbf{y}_{3}$ and $\left(x_{1}+e_{1}, x_{2}, x_{3}\right) \in \mathcal{E}_{1}$ can be connected via nearest-neighbor bonds in $\mathcal{E}_{1}$. Thus we have $\left\langle\left[G\left(\mathbf{x}_{3}\right)-G\left(x_{1}+e_{1}, x_{2}, x_{3}\right)\right]^{2}\right\rangle \leq C\langle g(-\mathcal{S}) g\rangle$. A similar inequality can be checked if $\left(x_{1}+e_{1}, x_{2}, x_{3}\right) \notin \mathcal{E}_{1}$. and this proves $(4.4)$.

Since $g\left(\mathbf{x}_{3}\right)=G\left(\mathbf{x}_{3}\right)$ on $\mathcal{E}_{1}$ and 0 otherwise, it is clear that $\left\langle 1_{\left\{\left|x_{1}-x_{3}\right|+\left|x_{3}-x_{2}\right| \leq R\right\}} g^{2}\right\rangle \leq$ $\left\langle 1_{\left\{\left|x_{1}-x_{3}\right|+\left|x_{3}-x_{2}\right| \leq R\right\}} G^{2}\right\rangle$. Thus to prove the lemma it will suffice to prove that

$$
\left\langle 1_{\left\{\left|x_{1}-x_{3}\right|+\left|x_{3}-x_{2}\right| \leq R\right\}} G^{2}\right\rangle \leq C(\lambda)\langle G,(\lambda-\Delta) G\rangle
$$

We can drop the part of $\Delta$ in the $x_{3}$ direction, making the right hand side smaller. Hence it is enough to prove that

$$
\left\langle 1_{\left\{\left|x_{1}\right|+\left|x_{2}\right| \leq R\right\}} G^{2}\right\rangle \leq C(\lambda)\langle G,(\lambda-\Delta) G\rangle
$$


for functions $G\left(x_{1}, x_{2}\right)$. Call $V=1_{\left\{\left|x_{1}\right|+\left|x_{2}\right| \leq R\right\}}$. It is local and bounded. We are in $\mathbb{Z}^{2 d}$ and we want to show that there is a $C(\lambda)$ such that $-C(\lambda)(\lambda-\Delta) \geq V$ as operators, or, equivalently

$$
V^{1 / 2}(\lambda-\Delta)^{-1} V^{1 / 2} \leq C(\lambda) .
$$

Let $G_{\lambda}(x, y)$ be the kernel of $(\lambda-\Delta)^{-1}$ and $\varphi: \mathbb{Z}^{2 d} \rightarrow \mathbb{R}$. Since $V$ is bounded by 1 , $\sum_{x, y \in \mathbb{Z}^{2 d}} V^{1 / 2}(x) G_{\lambda}(x, y) V^{1 / 2}(y) \varphi(x) \varphi(y)$ is easily bounded by $G_{\lambda}(0,0) \sum_{x} \varphi^{2}(x)$. If $d=1$, we are in $\mathbb{Z}^{2}$ and $C(\lambda)=G_{\lambda}(0,0) \leq C|\log \lambda|$. In $d=2$, we are in the transient case $\mathbb{Z}^{4}$ and $G_{\lambda}(0,0)$ is bounded uniformly in $\lambda$ (see [13]).

We now divide the complement of $\mathcal{E}_{1}$ into two sets. We call a site $x$ an isolated double site if

$$
x_{i}=x_{j}=x, \quad\left|x_{k}-x\right| \geq 5 \text { for all } k \neq i, j .
$$

We use here the lattice distance for $x, y \in \mathbb{Z}^{d}:|x-y|=\sum_{j=1}^{d}\left|x^{j}-y^{j}\right|$. Denote by $\mathcal{E}_{2}$ the set with at most isolated double sites and $\mathcal{E}_{3}=\left[\mathbb{Z}^{d}\right]^{n}-\left(\mathcal{E}_{1} \cup \mathcal{E}_{2}\right)$ the rest. For a configuration $\left(x_{1}, x_{1}, \cdots, x_{k}, x_{k}, x_{2 k+1}, x_{2 k+2} \cdots, x_{n}\right)$ with $k$ isolated double sites, we define $F=\mathbf{T} f$ by

$$
\begin{aligned}
& F\left(x_{1}, x_{1}, \ldots, x_{k}, x_{k}, x_{2 k+1}, x_{2 k+2} \ldots, x_{n}\right) \\
& =\underset{\mathbf{y}_{k}:\left|x_{i}-y_{i}\right|=1 \text { for all } i}{\operatorname{Av}} f\left(x_{1}, y_{1}, \ldots, x_{k}, y_{k}, x_{2 k+1}, \ldots, x_{n}\right)
\end{aligned}
$$

If $\mathbf{x} \in \mathcal{E}_{3}$, then $F(\mathbf{x})=0$, e.g., $F\left(x, x, x+e_{1}, x_{4}, \ldots, x_{n}\right)=0$. We also define the restriction $\mathbf{R} F$ by $\mathbf{R} F\left(\mathbf{x}_{n}\right)=F\left(\mathbf{x}_{n}\right)$ if $\mathbf{x}_{n} \in \mathcal{E}_{1}$ and $\mathbf{R} F(\mathbf{x})=0$ otherwise. Note that $\mathbf{T}$ and $\mathbf{R}$ are not inverse to each other although $R T$ is the identity.

Lemma 4.3 There is a $C(\lambda)$ as in (3.4) such that for any symmetric function $f$ on $\mathbb{Z}^{n}$ vanishing on $\mathcal{E}_{1}^{C}$ and $F=\mathbf{T} f$

$$
(1 / C(\lambda)) n^{-2}\langle F,(\lambda-\Delta) F\rangle \leq\langle f,(\lambda-\mathcal{S}) f\rangle \leq C\langle F,(\lambda-\Delta) F\rangle
$$

Define $\tilde{F}=\mathbf{T} \mathbf{R} F$ where $\mathbf{R}$ is the restriction. Then in addition,

$$
\langle F,(\lambda-\Delta) F\rangle \geq C(\lambda) n^{-2}\langle\tilde{F},(\lambda-\Delta) \tilde{F}\rangle
$$

Proof: By definition, $\langle F,-\Delta F\rangle$ is given by $\frac{1}{2} \sum_{\mathbf{x}, \mathbf{y}:|\mathbf{x}-\mathbf{y}|=1}[F(\mathbf{x})-F(\mathbf{y})]^{2}$. The upper bound of (4.6) is immediate, since $\mathcal{S}$ has Neumann boundary conditions, corresponding to dropping terms in the Dirichlet form with either $x$ or $y$ in $\mathcal{E}_{1}^{C}$. We now prove the lower bound in (4.6). We decompose $\mathbf{x}$ and $\mathbf{y}$ into three sets, $\mathcal{E}_{1}, \mathcal{E}_{2}, \mathcal{E}_{3}$, so that

$$
\langle F,-\Delta F\rangle=\sum_{\alpha=1,2,3 ; \beta=1,2,3} \Phi_{\alpha, \beta}
$$

where

$$
\Phi_{\alpha, \beta}=\frac{1}{2} \sum_{\mathbf{x} \in \mathcal{E}_{\alpha}, \mathbf{y} \in \mathcal{E}_{\beta}:|\mathbf{x}-\mathbf{y}|=1}[F(\mathbf{x})-F(\mathbf{y})]^{2} .
$$

If both $\mathbf{x}$ and $\mathbf{y}$ satisfy the hard core condition, then the contribution is

$$
\Phi_{1,1}=\frac{1}{2} \sum_{\mathbf{x}, \mathbf{y}:|\mathbf{x}-\mathbf{y}|=1}[f(\mathbf{x})-f(\mathbf{y})]^{2} \leq\langle f,-\mathcal{S} f\rangle .
$$


We can estimate terms where either $\mathbf{x}$ or $\mathbf{y}$ is in $\mathcal{E}_{3}$ by,

$$
\left|\Phi_{1,3}+\Phi_{2,3}+\Phi_{3,3}\right| \leq C \sum_{i \neq j \neq k}\left\langle 1_{\left\{\left|x_{i}-x_{k}\right|+\left|x_{k}-x_{j}\right| \leq R\right\}} F^{2}\right\rangle
$$

From the definition of $F$, we can check that

$$
\sum_{i \neq j \neq k}\left\langle 1_{\left\{\left|x_{i}-x_{k}\right|+\left|x_{k}-x_{j}\right| \leq R\right\}} F^{2}\right\rangle \leq C \sum_{i \neq j \neq k}\left\langle 1_{\left\{\left|x_{i}-x_{k}\right|+\left|x_{k}-x_{j}\right| \leq R\right\}} f^{2}\right\rangle
$$

The last term is bounded by $C(\lambda) n^{2}\langle f,(\lambda-\mathcal{S}) f\rangle$ from Lemma 4.2. Thus we have

$$
\left|\Phi_{1,3}+\Phi_{2,3}+\Phi_{3,3}\right| \leq C(\lambda) n^{2}\langle f,(\lambda-\mathcal{S}) f\rangle .
$$

We now bound $\Phi_{1,2}$. In this case we have, for example, $\mathbf{x}=\left(x_{1}, x_{1}+e_{1}, x_{3}, \cdots, x_{n}\right)$ and $\mathbf{y}=\left(x_{1}, x_{1}, x_{3}, \cdots, x_{n}\right)$. Notice that because $\mathbf{x} \in \mathcal{E}_{1}, \mathbf{y}$ can in fact have at most one double site. By assumption of isolated double sites, we have $\left|x_{j}-x_{1}\right| \geq 5$ for all $j \geq 3$. Thus

$$
F(\mathbf{y})=\underset{\left|z-x_{1}\right|=1}{\operatorname{Av}} f\left(x_{1}, z, x_{3}, \cdots, x_{n}\right)
$$

Under the assumption $\left|x_{j}-x_{1}\right| \geq 5$ for all $j \geq 3$ we can always connect $z$ to $x_{1}$. By Schwarz's inequality, we then have

$$
\sum_{x_{1}, x_{3}, \cdots, x_{n}}\left|f\left(x_{1}+e_{1}, z, x_{3}, \cdots, x_{n}\right)-f\left(x_{1}, z, x_{3}, \cdots, x_{n}\right)\right|^{2} \leq C\langle f,(-\mathcal{S}) f\rangle
$$

Hence

$$
\left|\Phi_{1,2}\right| \leq C\langle f,(-\mathcal{S}) f\rangle \text {. }
$$

Finally we bound $\Phi_{2,2}$. The typical case looks like $\mathbf{x}=\left(x_{1}, x_{1}, x_{3}, x_{3}+e_{1}, x_{5}, \cdots, x_{n}\right)$ and $\mathbf{y}=\left(x_{1}, x_{1}, x_{3}, x_{3}, x_{5}, \cdots, x_{n}\right)$. Then

$$
\begin{aligned}
& F\left(x_{1}, x_{1}, x_{3}, x_{3}+e_{1}, x_{5}, \cdots, x_{n}\right)-F\left(x_{1}, x_{1}, x_{3}, x_{3}, x_{5}, \cdots, x_{n}\right) \\
& =\underset{\left|z-x_{1}\right|=1}{\operatorname{Av}} \operatorname{Av}_{\left|w-x_{3}\right|=1}\left[f\left(x_{1}, z, x_{3}, x_{3}+e_{1}, x_{5}, \cdots, x_{n}\right)-f\left(x_{1}, z, x_{3}, w, x_{5}, \cdots, x_{n}\right)\right]
\end{aligned}
$$

Using Jensen's inequality and the same arguments as in the estimate of $\Phi_{1,2}$ above we obtain $\left|\Phi_{2,2}\right| \leq C\langle f,(-\mathcal{S}) f\rangle$. Putting all these estimates together, we have the lower bound of (4.6).

To prove (4.7), call $f=\mathbf{R} F$ so that $\tilde{F}=\mathbf{T} f$. From (4.6) we have $\langle f,(\lambda-\mathcal{S}) f\rangle \geq$ $C(\lambda) n^{-2}\langle\tilde{F},(\lambda-\Delta) \tilde{F}\rangle$. Since $\mathcal{S}$ is an operator with Neumann boundary condition, for any $F$ with $\mathbf{R} F=f$ we have $\langle F,(\lambda-\Delta) F\rangle \geq C\langle f,(\lambda-\mathcal{S}) f\rangle$ and this proves (4.7).

Lemma 4.4 Suppose that $f, g \in \mathcal{C}_{n}$. Let $F, G=\mathbf{T} f, \mathbf{T} g$. There is a $C(\lambda)$ as in (3.4) such that

$$
|\langle g, \mathcal{A} f\rangle-\langle G, A F\rangle| \leq C(\lambda) n^{1 / 2}\langle G,(\lambda-\Delta) G\rangle^{1 / 2}\langle F,(-\Delta) F\rangle^{1 / 2}
$$

Recall $\tilde{F}=$ T R F. We have

$$
|\langle A \tilde{F}, G\rangle-\langle A F, G\rangle| \leq C(\lambda) n^{1 / 2}\langle G,(\lambda-\Delta) G\rangle^{1 / 2}\langle F,(-\Delta) F\rangle^{1 / 2}
$$

where $C$ is independent of $\lambda$. 
Proof: We first prove (4.8). Note that it suffices to prove it with $\mathcal{A}$ replaced by $\mathcal{A}_{+}$, since it then follows for $\mathcal{A}_{+}^{*}$ and thus for $\mathcal{A}=\mathcal{A}_{+}-\mathcal{A}_{+}^{*}$. Suppose first of all that $f \in \mathcal{M}_{n}$. Recall the definition of $\mathcal{A}_{+}$in (2.8). It suffices to consider one term in the summation, say $\mathcal{A}_{+}^{i j} f=\delta\left(x_{2}-x_{1}-e_{1}\right) \prod_{k \neq 2}\left(1-\delta\left(x_{2}-x_{k}\right)\right) \nabla_{+}^{1,2} f$ and $A_{+}^{i j} f=\delta\left(x_{2}-x_{1}-e_{1}\right) \nabla_{+}^{1,2} f$. We divide $\mathbb{Z}^{(n+1) d}$ into $\mathcal{N}=\left\{\left|x_{j}-x_{1}\right|>5\right.$ for all $\left.j \geq 3\right\}$. and its complement. If $\mathbf{x}_{n+1} \in \mathcal{N}$, then $\left(A_{+}^{12} F-\mathcal{A}_{+}^{12} f\right)\left(\mathbf{x}_{n+1}\right)=0$ so we can estimate

$$
\left|\left\langle g, 1_{\mathcal{N}^{C} \mathcal{A}} \mathcal{A}_{+}^{12} f\right\rangle\right|^{2} \leq\left\|f\left(x_{1}+e_{1}, x_{3} \cdots, x_{n+1}\right)-f\left(x_{1}, x_{3} \cdots, x_{n+1}\right)\right\|^{2}\left\|g 1_{\mathcal{N}^{C}}\right\|^{2}
$$

Clearly, $1_{\mathcal{N}^{C}} \leq \sum_{j \geq 3}\left\{\left|x_{j}-x_{1}\right| \leq 5\right\}$. By Lemma 4.2,

$$
\left\|g 1_{\mathcal{N} C}\right\|^{2} \leq C(\lambda) n^{2}\langle g,(\lambda-\mathcal{S}) g\rangle .
$$

Replacing 1,2 by $i, j$ and summing over all $i, j$, by the permutation symmetry of $f$ we have

$$
\left|\left\langle g, 1_{\mathcal{N}^{C}} \mathcal{A}_{+} f\right\rangle\right|^{2} \leq C(\lambda) n^{2}\langle g,(\lambda-\mathcal{S}) g\rangle n^{-1}\langle f,(-\mathcal{S}) f\rangle
$$

A similar bound holds for $F$ on $\mathcal{N}^{c}$. Combining these estimates and using Lemma 4.3, we obtain (4.8) for $\mathcal{A}_{+}$.

If $f \in \mathcal{C}_{n}$, write $f=\left(f_{1}, \ldots, f_{n}, 0,0, \ldots\right)$. From (4.11) we have

$$
\left|\left\langle g_{(k+1)}, \mathcal{A}_{+} f_{(k)}\right\rangle\right| \leq C(\lambda) n^{1 / 2} \varepsilon\left\langle g_{(k+1)},(\lambda-\mathcal{S}) g_{(k+1)}\right\rangle+C n^{1 / 2} \varepsilon^{-1}\left\langle f_{(k)},(-\mathcal{S}) f_{(k)}\right\rangle
$$

Summing over $k$ and optimizing $\varepsilon$, we get (4.8). Repeating the proof, i.e., noting that $A_{+}^{12} F-A_{+}^{12} \tilde{F}=0$ on $\mathcal{N}$ and using Lemma 4.2, to bound the term on $\mathcal{N}^{C}$, gives (4.9).

Lemma 4.5 There is a $C(\lambda)$ as in (3.4) such that for all $f \in \mathcal{C}_{n}$, if $F=\mathbf{T} f$

$$
\begin{aligned}
C^{-1} & \left\langle A F,(\lambda-\Delta)^{-1} A F\right\rangle-C^{2}(\lambda) n\langle F,(\lambda-\Delta) F\rangle \\
\quad \leq & \left\langle\mathcal{A} f,(\lambda-\mathcal{S})^{-1} \mathcal{A} f\right\rangle \\
& \leq C(\lambda) n^{2}\left\langle A F,(\lambda-\Delta)^{-1} A F\right\rangle+C^{3}(\lambda) n^{3}\langle F,(\lambda-\Delta) F\rangle .
\end{aligned}
$$

Recall $\tilde{F}=\mathbf{T} \mathbf{R} F$. Then we have

$$
\left\langle A F,(\lambda-\Delta)^{-1} A F\right\rangle \geq C^{-1}\left\langle A \tilde{F}(\lambda-\Delta)^{-1} A \tilde{F}\right\rangle-C^{2}(\lambda) n\langle F,(\lambda-\Delta) F\rangle
$$

Proof: Recall the variational formula

$$
\left\langle\mathcal{A} f,(\lambda-\mathcal{S})^{-1} \mathcal{A} f\right\rangle=\sup _{g}\{2\langle g, \mathcal{A} f\rangle-\langle g,(\lambda-\mathcal{S}) g\rangle\}
$$

By Lemmas 4.3 and 4.4, we can bound the right side from above by

$$
\begin{aligned}
& 2\langle g, \mathcal{A} f\rangle-\langle g,(\lambda-\mathcal{S}) g\rangle \\
& \leq 2\langle G, A F\rangle-(1 / C(\lambda)) n^{-2}\langle G,(\lambda-\Delta) G\rangle+C(\lambda) n^{1 / 2}\langle G,(\lambda-\Delta) G\rangle^{1 / 2}\langle F,(\lambda-\Delta) F\rangle^{1 / 2} \\
& \leq C(\lambda) n^{2}\left\langle A F,(\lambda-\Delta)^{-1} A F\right\rangle+C^{3}(\lambda) n^{3}\langle F,(\lambda-\Delta) F\rangle
\end{aligned}
$$

which give the upper bound in (4.12). Alternatively we can bound the right side below by

$$
\begin{aligned}
& 2\langle g, \mathcal{A} f\rangle-\langle g,(\lambda-\mathcal{S}) g\rangle \\
& \geq 2\langle G, A F\rangle-C(\lambda) n^{1 / 2}\langle G,(\lambda-\Delta) G\rangle^{1 / 2}\langle F,(\lambda-\Delta) F\rangle^{1 / 2}-C\langle G,(\lambda-\Delta) G\rangle \\
& \geq 2\langle G, A F\rangle-C\langle G,(\lambda-\Delta) G\rangle-C^{2}(\lambda) n\langle F,(\lambda-\Delta) F\rangle
\end{aligned}
$$


Optimizing over $G$,

$$
2\langle g, \mathcal{A} f\rangle-\langle g,(\lambda-\mathcal{S}) g\rangle \geq C\left\langle A F,(\lambda-\Delta)^{-1} A F\right\rangle-C^{2}(\lambda) n\langle F,(\lambda-\Delta) F\rangle
$$

This proves the lower bound in (4.12). By definition,

$$
\left\langle A F,(\lambda-\Delta)^{-1} A F\right\rangle=\sup _{G}\{2\langle A F, G\rangle-\langle G(\lambda-\Delta) G\rangle\}
$$

By Lemma 4.4, the right hand side of (4.14) is bounded by

$$
\begin{aligned}
& 2\langle A F, G\rangle-\langle G(\lambda-\Delta) G\rangle \\
& \geq 2\langle A \tilde{F}, G\rangle-C(\lambda) n^{1 / 2}\langle G,(\lambda-\Delta) G\rangle^{1 / 2}\langle F,(\lambda-\Delta) F\rangle^{1 / 2}-\langle G,(\lambda-\Delta) G\rangle \\
& \geq 2\langle A \tilde{F}, G\rangle-C\langle G,(\lambda-\Delta) G\rangle-C^{2}(\lambda) n\langle F,(\lambda-\Delta) F\rangle .
\end{aligned}
$$

Taking the sup over $G$, we prove 4.13).

Our interest is in the inner product $\langle\langle\rangle$,$\rangle . It is easy to check that all previous Lemmas$ hold for the inner product $\langle\langle\rangle$,$\rangle as well. Since extensions from \langle$,$\rangle to \langle\langle\rangle$,$\rangle were carried out in$ detail in [8], we shall only outline the basic procedures to prove these Lemmas for the inner product $\langle\langle$,$\rangle .$

We now prove the analogue of Lemma 4.3 for the inner product $\langle\langle\rangle$,$\rangle . For any local$ functions we rewrite the inner product as

$$
\langle\langle g, h\rangle\rangle=\lim _{k \rightarrow \infty}(2 k+1)^{-2}\left\langle\sum_{|x| \leq k} \tau_{x} g ; \sum_{|x| \leq k} \tau_{x} h\right\rangle
$$

Similarly, we have

$$
\langle\langle g,(-\mathcal{S}) h\rangle\rangle=\lim _{k \rightarrow \infty}(2 k+1)^{-2}\left\langle\sum_{|x| \leq k} \tau_{x} g ;(-\mathcal{S}) \sum_{|x| \leq k} \tau_{x} h\right\rangle
$$

Recall the definition of $F=\mathbf{T} f$ is linear in $f$. Furthermore, the intersection properties of $\left(x_{1}, \cdots, x_{n}\right)$ are independent of the translation, i.e. $\mathbf{x} \in \mathcal{E}_{i}$ iff $\tau_{z} \mathbf{x} \in \mathcal{E}_{i}$. Thus we have

$$
\mathbf{T} \sum_{|x| \leq k} \tau_{x} h=\sum_{|x| \leq k} \tau_{x} \mathbf{T} h
$$

For each fixed $k$ everything is still local so by Lemma 4.3,

$$
(2 k+1)^{-2}\left\langle\sum_{|x| \leq k} \tau_{x} F,(\lambda-\Delta) \sum_{|x| \leq k} \tau_{x} F\right\rangle \leq C(\lambda) n^{2}(2 k+1)^{-2}\left\langle\sum_{|x| \leq k} \tau_{x} f,(\lambda-\mathcal{S}) \sum_{|x| \leq k} \tau_{x} f\right\rangle
$$

Lettting $k \rightarrow \infty$ limit and using that the limits exist on both side we obtain Lemma 4.3 for $\langle\langle\cdot, \cdot\rangle\rangle$. To prove lemmas 4.4 and 4.5 for $\langle\langle\cdot, \cdot\rangle\rangle$, we only have to use that $A$ commutes with translations.

Proof of Lemma 3.1 Upper Bound: We start with the variational formula (see (2.16)),

$$
\left\langle\left\langle w,\left(\lambda-\mathcal{L}_{n}\right)^{-1} w\right\rangle\right\rangle=\sup _{f \in \mathcal{C}_{n}}\left\{2\langle\langle w, f\rangle\rangle-\left\langle\left\langle\left(\lambda-\mathcal{L}_{n}\right) f,(\lambda-\mathcal{S})^{-1}\left(\lambda-\mathcal{L}_{n}\right) f\right\rangle\right\rangle\right\}
$$


where $\mathcal{C}_{n}$ denotes the functions of degree less than or equal to $n$. By definition,

$$
\left\langle\left\langle\left(\lambda-\mathcal{L}_{n}\right) f,(\lambda-\mathcal{S})^{-1}\left(\lambda-\mathcal{L}_{n}\right) f\right\rangle\right\rangle=\langle\langle f,(\lambda-\mathcal{S}) f\rangle\rangle+\left\langle\left\langle\mathcal{A} f,(\lambda-\mathcal{S})^{-1} \mathcal{A} f\right\rangle\right\rangle
$$

Here we have set $\mathcal{A}_{+}=0$ on $\mathcal{M}_{n}$ since we are considering only $\mathcal{L}_{n}$. By Lemmas 4.3 and 4.5 ,

$$
\begin{aligned}
\langle\langle f,(\lambda-\mathcal{S}) f\rangle\rangle+\left\langle\left\langle\mathcal{A} f,(\lambda-\mathcal{S})^{-1} \mathcal{A} f\right\rangle\right\rangle \geq & \left(n^{-2} / C(\lambda)\right)\langle\langle F,(\lambda-\Delta) F\rangle\rangle \\
& +n^{-2} C(\lambda)\left\langle\left\langle A F,(\lambda-\Delta)^{-1} A F\right\rangle\right\rangle .
\end{aligned}
$$

Since $\langle\langle f, w\rangle\rangle$ and $\langle\langle F, w\rangle\rangle$ vanish except for functions of degree 2 , it is easy to check that $\langle\langle f, w\rangle\rangle=\langle\langle F, w\rangle$. Thus we have

$$
\begin{aligned}
& \left\langle\left\langle w,\left(\lambda-\mathcal{L}_{n}\right)^{-1} w\right\rangle\right\rangle \\
& \leq \sup _{F=\mathbf{T} f}\left\{2\langle\langle F, w\rangle\rangle-(1 / C(\lambda)) n^{-2}\left\langle\left\langle A F,(\lambda-\Delta)^{-1} A F\right\rangle\right\rangle-C(\lambda) n^{-2}\langle\langle F,(\lambda-\Delta) F\rangle\rangle\right\} \\
& \leq C(\lambda) n^{2}\left\langle\left\langle w,\left(\lambda-L_{n}\right)^{-1} w\right\rangle\right\rangle .
\end{aligned}
$$

Lower Bound: We have

$$
\left\langle\left\langle w,\left(\lambda-L_{n}\right)^{-1} w\right\rangle\right\rangle=\sup _{F}\left\{2\langle\langle F, w\rangle\rangle-\left\langle\left\langle A F,(\lambda-\Delta)^{-1} A F\right\rangle\right\rangle-\langle\langle F,(\lambda-\Delta) F\rangle\rangle\right\}
$$

Since $\langle\langle\tilde{F}, w\rangle\rangle=\langle\langle F, w\rangle\rangle$, from Lemma 4.5 we have

$$
\left\langle\left\langle w,\left(\lambda-L_{n}\right)^{-1} w\right\rangle\right\rangle \leq \sup _{F}\left\{2\langle\langle\tilde{F}, w\rangle\rangle-C^{-1}\langle\langle F,(\lambda-\Delta) F\rangle\rangle-\frac{n^{-1}}{C^{2}(\lambda)}\left\langle\left\langle A \tilde{F},(\lambda-\Delta)^{-1} A \tilde{F}\right\rangle\right\rangle\right\} .
$$

By Lemma 4.3, $\langle\langle F,(\lambda-\Delta) F\rangle\rangle \geq C(\lambda) n^{-2}\langle\langle\tilde{F},(\lambda-\Delta) \tilde{F}\rangle\rangle$. Recall $\mathbf{R} F=f, \tilde{F}=\mathbf{T} f$. Thus the previous line is bounded above by

$$
\sup _{f=\mathbf{R} F}\left\{2\langle\langle\mathbf{T} f, w\rangle\rangle-\frac{1}{C(\lambda)} n^{-2}\langle\langle\mathbf{T} f,(\lambda-\Delta) \mathbf{T} f\rangle\rangle-\frac{1}{C^{2}(\lambda)} n^{-1}\left\langle\left\langle A \mathbf{T} f,(\lambda-\Delta)^{-1} A \mathbf{T} f\right\rangle\right\rangle\right\}
$$

Clearly, $\langle\langle\mathbf{T} f, w\rangle\rangle=\langle\langle f, w\rangle\rangle$. By Lemma 4.3, we have $\langle\langle\mathbf{T} f,(\lambda-\Delta) \mathbf{T} f\rangle\rangle \geq C\langle\langle f,(\lambda-\mathcal{S}) f\rangle\rangle$. By Lemma 4.5, we have

$$
\begin{aligned}
\left\langle\left\langle w,\left(\lambda-L_{n}\right)^{-1} w\right\rangle\right\rangle & \leq 2\langle\langle f, w\rangle\rangle-C^{-1} n^{-2}\langle\langle f,(\lambda-\mathcal{S}) f\rangle\rangle-(1 / C(\lambda)) n\left\langle\left\langle\mathcal{A} f,(\lambda-\mathcal{S})^{-1} \mathcal{A} f\right\rangle\right\rangle \\
& \leq C(\lambda) n^{2}\left\langle\left\langle w,\left(\lambda-\mathcal{L}_{N}\right)^{-1} w\right\rangle\right\rangle
\end{aligned}
$$

This proves the Lemma.

Acknowledgement: H.T.Yau would like to thank P. Deift, J. Baik and H. Spohn for explaining their results. In particular, Spohn has pointed out the relation (1.1) so that the connection between the current across the zero and the diffusion coefficient becomes transparent. H.T.Yau would also like to thank A. Sznitman for his hospitality and invitation to lecture on this subject at ETH. 


\section{References}

[1] J. Baik, P.A. Deift, K. Johansson, On the distribution of the length of the longest increasing subsequence in a random permutation. J. Amer. Math. Soc. 12, 1119-1178 (1999)

[2] J. Baik, E.M. Rains: Limiting distributions for a polynuclear growth model with external sources. J. Stat. Phys. 100, 523-542 (2000)

[3] H. van Beijeren, R. Kutner, H. Spohn: Excess noise for driven diffusive systems. Phys. Rev. Lett. 54, 2026-2029 (1985)

[4] E.B. Davies, Heat Kernels and Spectral Theory, Cambridge U. Press, 1989.

[5] K. Johansson: Shape fluctuations and random matrices. Comm. Math. Phys. 209, 437476 (2000)

[6] M. Kardar, G. Parisi, Y.C.Zhang, Dynamical scaling of growing interfaces. Phys. Rev. Lett. 56 , 889-892.

[7] Landim, C.; Olla, S.; Yau, H. T: Some properties of the diffusion coefficient for asymmetric simple exclusion processes. Ann. Probab. 24 (1996), no. 4, 1779-1808.

[8] Landim, C.; Yau, H. T. Fluctuation-dissipation equation of asymmetric simple exclusion processes. Probab. Theory Related Fields 108 (1997), no. 3, 321-356.

[9] T. Liggett, Interacting Particle Systems, Springer-Verlag, New York, 1985.

[10] M. Prähofer, H. Spohn: Current Fluctuations for the Totally Asymmetric Simple Exclusion Process, preprint cond-mat/0101200 v2.

[11] M. Prähofer, H. Spohn, Universal distribution for growth processes in $1+1$ dimensions and random matrices. Phys. Rev. Lett. 84, 4882-4885 (2000)

[12] Sethuraman, S.; Varadhan, S. R. S.; Yau, H.T. : Diffusive limit of a tagged particle in asymmetric simple exclusion processes. Comm. Pure Appl. Math. 53 (2000), no. 8, $972-1006$.

[13] F. Spitzer, Principles of Random Walks, Springer-Verlag, New York, 1976.

[14] Spohn, H: "Large Scale Dynamics of Interacting Particles", Springer-Verlag, New York, 1991. 